\title{
Effect of high versus low ambient humidity on the severity of obstructive sleep apnoea
}

\author{
Ruzica Jokic, Lal Bhagchandani, Trevor Zintel, Marilyn Baetz, Michael F Fitzpatrick
}

\begin{abstract}
Background-Surface tension forces appear to make a significant contribution to upper airway closure in patients with obstructive sleep apnoea (OSA). It is possible that drying of the upper airway mucosa at night might contribute to these surface tension forces and the severity of OSA might therefore change with alteration of the ambient humidity. Methods-A randomised single blind crossover study of high ambient relative humidity (HRH) versus low ambient relative humidity (LRH) was performed in 12 men of mean (SD) age 49 (9) years with mild OSA (apnoea/hypopnoea index (AHI) $14(5.2))$. On one night patients slept in continuous HRH (85 (4)\%, range $80-93 \%)$ and on the other in LRH (16 (4)\%, range $11-22 \%$ ).

Results-The AHI was similar on the HRH and LRH nights (mean difference 3; $95 \%$ CI -2 to $9, p=0.20$ ) and no statistically significant differences in AHI were observed on the two nights after standardising for body position and sleep stage. Sleep stage distribution and the proportion of time spent in the supine position were similar on the HRH and LRH nights. The number of nonrespiratory arousals was also similar on the two nights.

Conclusion-Altering ambient humidity alone has no significant impact on the severity of OSA.

(Thorax 1999;54:711-713)
\end{abstract}

Keywords: obstructive sleep apnoea; humidity; surface tension

Saskatchewan,

Saskatoon,

Saskatchewan, Canada

Correspondence to:

Dr M F Fitzpatrick, Division

of Respiratory and Critical

Care Medicine, Richardson

House, Queen's University,

Kingston, Ontario, Canada

K7L 3N6.

Received 16 October 1998

Returned to author

19 January 1999

Revised manuscript received

15 March 1999

Accepted for publication

11 May 1999

the of upper airway surface tension in the pathogenesis of obstructive sleep apnoea (OSA) has received relatively little attention to date. Data from several groups suggest that surface tension forces may play an important part in upper airway obstruction. We have shown that the addition of a topical lubricant to the upper airway, administered as nose drops, significantly reduced the severity of OSA. ${ }^{1}$ Others have shown that the addition of topical surfactant reduces the opening pressure of the canine $^{2}$ and awake human ${ }^{3}$ upper airway, and may even help prevent closure of the patent upper airway.

OSA tends to become more severe with the passage of time over night, irrespective of sleep stage or body position. ${ }^{4}$ We surmised that drying of upper airway secretions during the night, through increasing surface tension forces in the upper airway, might contribute to increasing severity of OSA. High inspired humidity, by reducing the surface tension of pharyngeal and hypopharyngeal mucus, might then reduce the severity of OSA. The volume of upper airway mucus lining the pharyngeal mucosa is small, and direct measurement of the surface tension of these secretions is thus extremely difficult in humans. The aim of this study was to evaluate whether alteration of ambient humidity would effect a clinically important change in the severity of upper airway obstruction in patients with OSA.

\section{Methods}

HYPOTHESIS

High ambient relative humidity will be associated with a lower apnoea/hypopnoea index (AHI) than low ambient relative humidity in patients with mild OSA.

\section{SUBJECTS}

Twelve men of mean age 48.6 (9.4) years and body mass index $30(4) \mathrm{kg} / \mathrm{m}^{2}$ with mild or moderate OSA defined as subjective daytime sleepiness accompanied by either an apnoea index of $>5$ or AHI of $>10$ were selected for study (mean (SD) AHI at the diagnostic study 14 (5)). All patients gave written informed consent. The University of Saskatchewan Advisory Committee on Ethics in Human Experimentation approved the research protocol and the consent form.

Patients with respiratory disorders, sleep disorders other than OSA, or fluid balance disorders, and patients taking medications that might interfere with hydration of the upper airway mucosa were excluded from the study.
STUDY DESIGN

The study was of a randomised, single blind, crossover design. Saskatchewan offers an ideal setting for this study because of very low indoor ambient relative humidity levels in winter. This allowed comparison of the effect of very low inspired humidity with an artificially created environment of high inspired humidity. 
Table 1 Sleep architecture and the duration of stage 2 non-REM sleep and REM sleep on the two study nights

\begin{tabular}{lccl}
\hline & High humidity & Low humidity & p value \\
\hline Wakefulness (\%TRT) & $17.5(2.5)$ & $17.1(2.6)$ & 0.90 \\
Stage 1 (\%TST) & $8.3(1.3)$ & $6.6(1.0)$ & 0.27 \\
Stage 2 (\%TST) & $50.0(2.5)$ & $48.2(2.0)$ & 0.64 \\
Slow wave sleep (\%TST) & $18.2(2.6)$ & $19.5(2.4)$ & 0.47 \\
REM sleep (\%TST) & $23.5(1.6)$ & $26.6(1.9)$ & 0.30 \\
Total sleep time (min) & $349(15.2)$ & $348(14.5)$ & 0.98 \\
Sleep efficiency (TST/TRT) & $80.6(2.4)$ & $81.2(2.6)$ & 0.82 \\
Stage 2 supine position (min) & $56(9)$ & $67(10)$ & 0.30 \\
Stage 2 lateral position (min) & $122(10)$ & $106(9)$ & 0.20 \\
REM lateral (min) & $68(7)$ & $70(6)$ & 0.82
\end{tabular}

TRT $=$ total recording time; TST $=$ total sleep time.

Patients spent two consecutive nights in the sleep laboratory. Each patient slept with the upper part of the body loosely enclosed in an identical plastic tent (model 1012 King Hut, Peace Medical, Orange, New Jersey, USA ) on both study nights. On one night highly humidified air (ultrasonic humidifier BT-201, Bionaire, Montreal, Canada) was pumped through the tent, and on the other night the tent was not connected to any outside humidity. Ambient relative humidity inside the tent was monitored continuously by a humidity probe located inside the tent (Humidity Indicator HMI 11, Vaisala Inc, Plain City, Ohio, USA) distant from the incoming airstream. The ambient temperature inside the tent was also continuously monitored (78214 C temperature meter, Hewlett Packard, Kirkland, Canada) and maintained at $24(1)^{\circ} \mathrm{C}$.

The humidification system we chose generated high relative humidity levels without excessive condensation in the tent; an outflow valve trapped the larger water droplets emitted from the ultrasonic humidifier and the loose fitting of the upper body tents allowed the highly humidified air to flow through the body tent into the room. The combination of an ultrasonic humidifier and variable air flow generation from an air compressor (Air Compressor System, Timeter, PCS-1, Leola, Pennsylvania, USA) facilitated maintenance of high relative humidity even when the patient changed position during sleep and the volume of humid air escaping from the tent increased. The relative humidity inside the body tent was

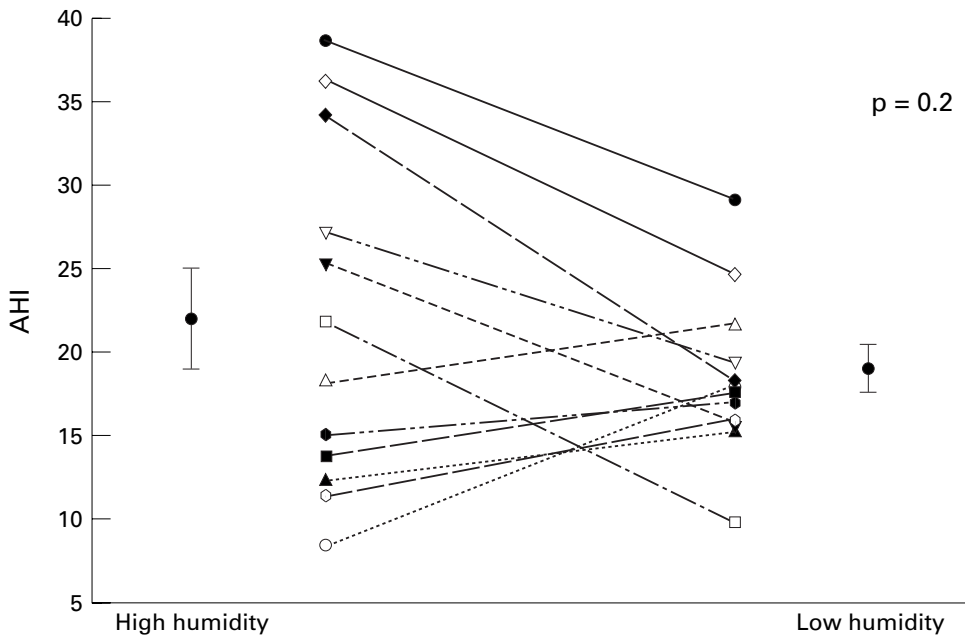

Figure 1 Severity of sleep apnoea on high and low humidity nights. maintained at or above $80 \%$ throughout the high humidity night.

Polysomnography included EEG, EOG, submental EMG, pulse oximetry, oronasal airflow, electrocardiogram, chest and abdominal movement, snore (vibration sensor), intercostal EMG and anterior tibialis EMG (Sandman Sleep System, Nellcor Puritan Bennett, Ottawa, Canada). Patients were continuously monitored by infrared video camera throughout the night. Sleep and arousals were scored according to conventional criteria. ${ }^{56}$

The single blind study design ensured that the individual scoring the sleep studies was unaware of the high or low humidity environment of the study.

\section{DATA ANALYSIS}

Two tailed paired $t$ testing was used to compare sleep and respiratory variables between the two nights.

We postulated that the overall difference in AHI between the low and high ambient humidity nights would be similar to that previously demonstrated between lubricant and placebo treatments in patients with mild to moderate OSA by Jokic et $a l^{1}$ - that is, at least 8. The standard deviation for the difference in AHI on high and low humidity nights was 8.2. Applying this information to the formula for calculation of statistical power ${ }^{7}$ revealed that 12 complete data sets would provide a statistical power of $90 \%$ to detect a true difference in AHI between the groups $(\alpha=0.05)$.

\section{Results}

The mean relative humidity level for all subjects on the high humidity night (HRH) was $85(4) \%$ (range $80-93 \%$ ), and on the low humidity night (LRH) was 16 (4)\% (range 11-22\%). No significant differences in sleep architecture, total sleep time, or the proportion of the total sleep time spent in the supine position or in the lateral position were observed between the two study nights (table 1).

The AHI was similar on the HRH (mean 22) and LRH nights (19; mean difference 3; 95\% CI -2 to $9, p=0.20$; fig 1 ). There were no statistically significant differences in AHI after standardising for body position (supine or lateral) and sleep stage (stage 2 non-REM sleep and REM sleep) between the HRH and LRH nights. The a priori minimum sleep requirement for this comparison was 30 minutes in a given sleep stage and body position on both nights.

Changing ambient humidity did not influence the duration of apnoeas and hypopnoeas, mean and minimum oxygen saturation (during both non-REM and REM sleep), or the frequency of non-respiratory arousals during sleep.

\section{Discussion}

This study shows that, contrary to our hypothesis, altering ambient humidity has no significant effect on the severity of OSA. The current study was undertaken for several reasons: (1) as a logical sequel to our recent finding ${ }^{1}$ that the severity of OSA is significantly reduced by 
application of a topical upper airway lubricant. The present study sought to answer whether simple humidification of the upper airway would have a similar effect to application of a topical lubricant; (2) to evaluate theoretical benefits of increased inspired humidity on upper airway patency ${ }^{138}$ in clinical practice; (3) previous studies have shown equivocal improvements in upper airway patency with moisturising the upper airway ${ }^{39}$ but these were experiments of short duration and none were conducted during natural sleep.

These negative findings suggest that the beneficial effect of adding topical phosphocholinamin to the upper airway ${ }^{1}$ is not simply an effect of moisturising the upper airway. The surfactant properties of phosphocholinamin would therefore appear more important than the addition of liquid to the airway surface in reducing the severity of upper airway obstruction.

The stability of airways is significantly influenced by the surface tension and fluid elasticity of mucosal secretions. ${ }^{10}$ However, little is known about the effects of moisture and surfactants on the physical properties of the mucus lining the supraglottic airway.

The addition of hypotonic solutions may produce reflex activation of upper airway dilator musculature, ${ }^{8}$ but the effect of saline (whether isotonic or hypotonic) on upper airway opening and closing was found to be small and inconsistent relative to the effect of applied surfactants. ${ }^{39}$ Application of saline to the supraglottic airway in anaesthetised dogs was associated with a significant reduction in upper airway resistance only during expiration. ${ }^{9}$ In five awake normal subjects a non-significant reduction in mean upper air- way opening pressure was reported after application of hypotonic saline, whereas a clear reduction in upper airway opening and closing pressures and increased upper airway diameter at any given level of pressure applied to the upper airway was found after application of surfactant. $^{3}$

In keeping with the literature mentioned above, the high ambient humidity in the current study had no significant clinical effect on upper airway closure in patients with mild OSA. We conclude therefore that altering ambient humidity alone does not effect a clinically important change in the severity of OSA.

This work was supported by the Heart and Stroke Foundation of Saskatchewan.

1 Jokic R, Klimaszevski A, Mink J, et al. Surface tension forces in sleep apnea: the role of a tissue lubricant. Am $\mathcal{F}$ Respir Crit Care Med 1998;157:1522-5.

2 Miki H, Hida W, Kikuchi Y, et al. Effects of pharyngeal lubrication on the opening of obstructed upper airway. 7 Appl Physiol 1995;72:2311-6.

3 Van der Touw T, Crawford ABH, Wheatley JR. Effects of a synthetic lung surfactant on pharyngeal patency in awake synthetic lung surfactant on pharyngeal paten
human subjects. $\mathcal{F}$ Appl Physiol 1997;82:78-85.

4 Charbonneau M, Martin JM, Olha A, et al. Changes in Charbonneau M, Martin JM, Olha A, et al. Changes in
obstructive sleep apnea characteristics through the night. obstructive sleep apnea char
Chest 1994;106:1695-701.

5 Rechtschaffen A, Kales A. A manual of standardized terminology: techniques and scoring system for sleep stages of human subjects. Los Angeles: BIS/BRI, University of California at Los Angeles, 1968: 1-15.

6 American Sleep Disorders Association. EEG arousals: scoring rules and examples. Sleep 1992;15:173-84.

7 Altman DG. Statistics and ethics in medical research. 3. How large a sample ? BMF 1980;281:1336-8.

8 Kuna ST, Sant'Ambrogio FB, Sant'Ambrogio G. Effect of airway surface liquid composition on laryngeal muscle activation. Sleep 1996;19(Suppl):S180-3.

9 Widdicombe JG, Davies A. The effects of a mixture of surface active agents (Sonarex) on upper airways resistance and snoring in anaesthetised dogs. Eur Respir f 1988;1: 785-91.

10 Hsu SH, Strohl KP, Jamieson AM. Role of viscoelasticity in tube model of airway reopening. 1. Non-Newtonian sols. $\mathcal{F}$ Appl Physiol 1994;76:2481-9. 